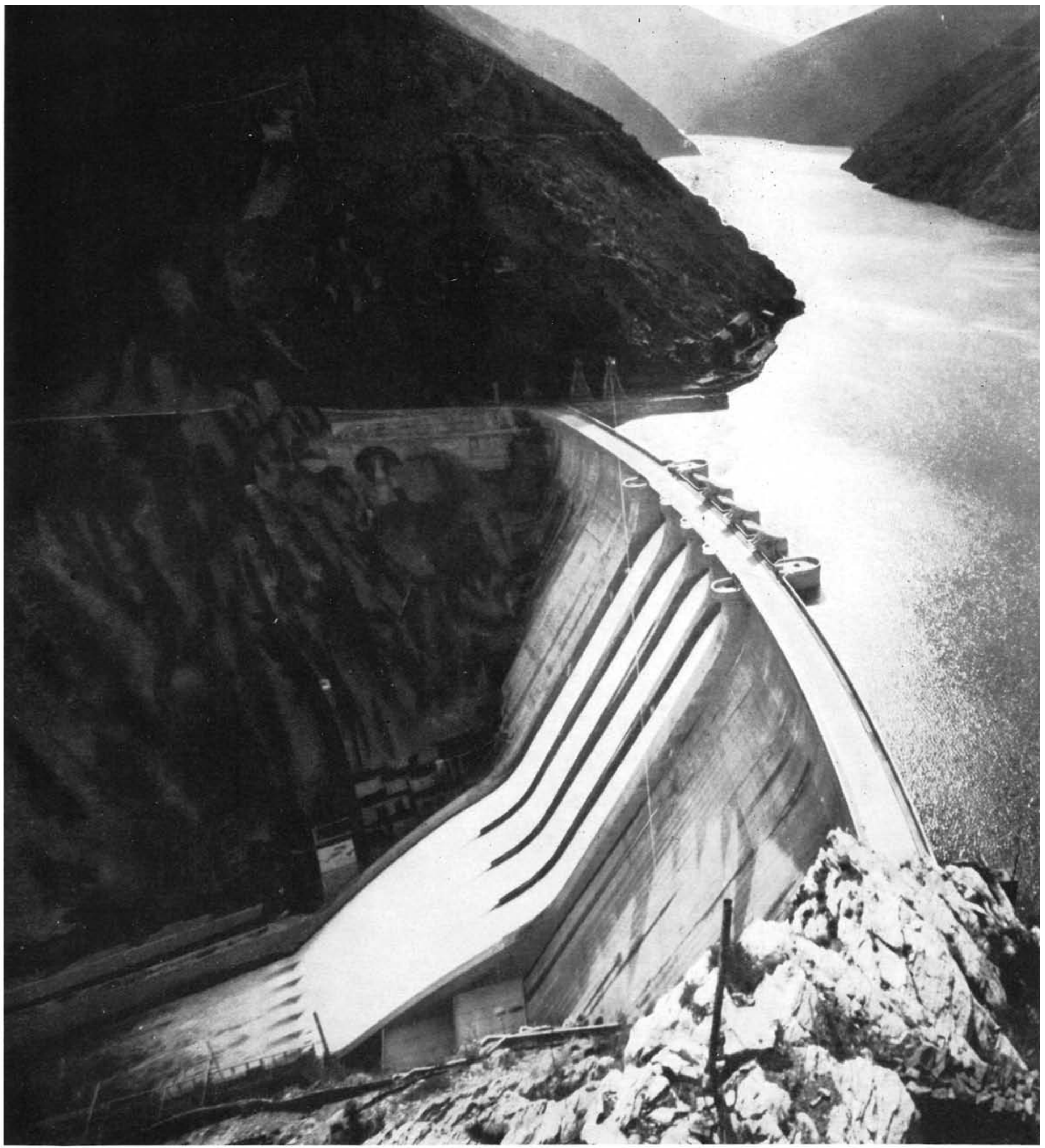

\title{
aprovechamiento hidroeléctrico
}



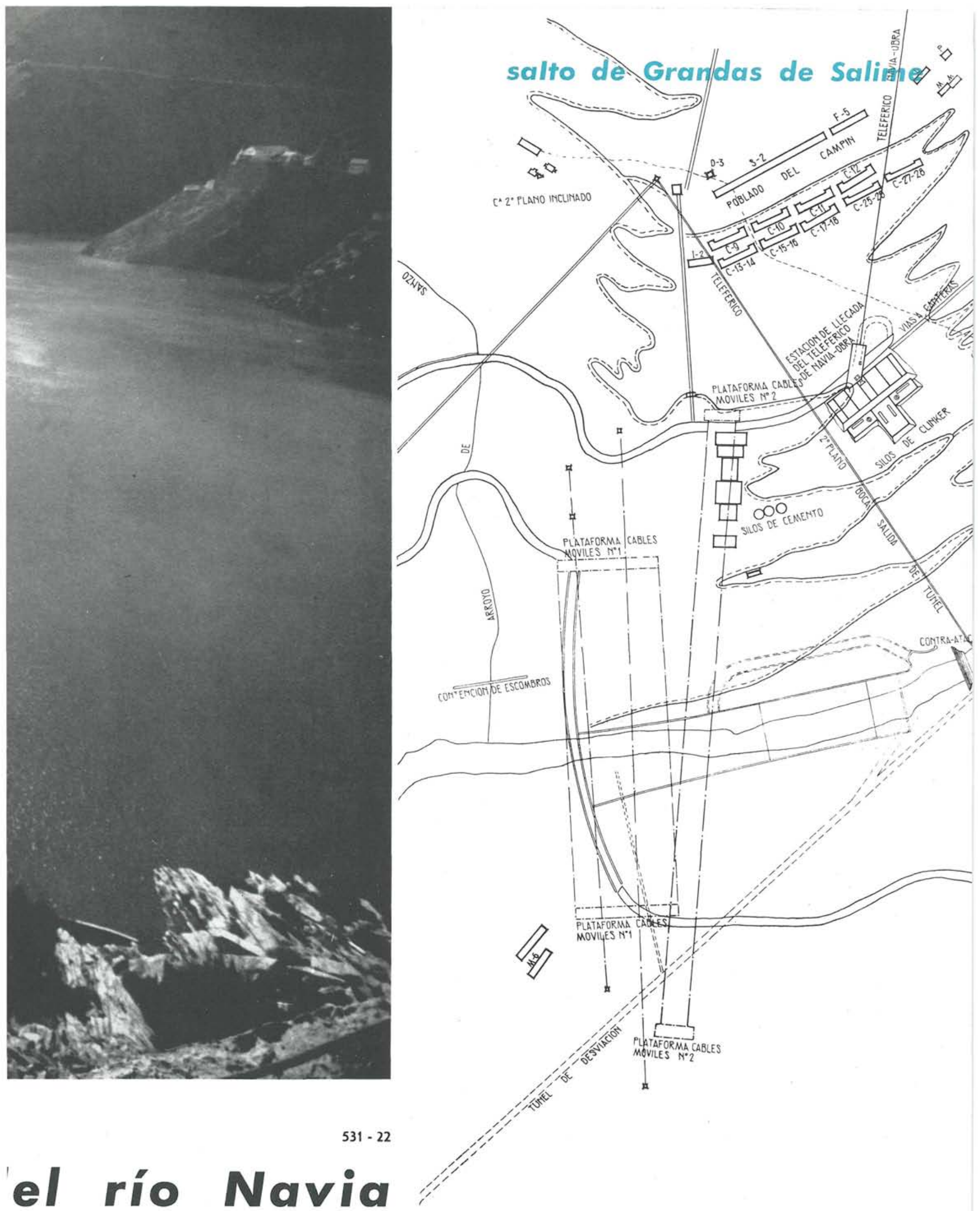

el río Navia

Fotografias: Pando-Madrid

planta general 


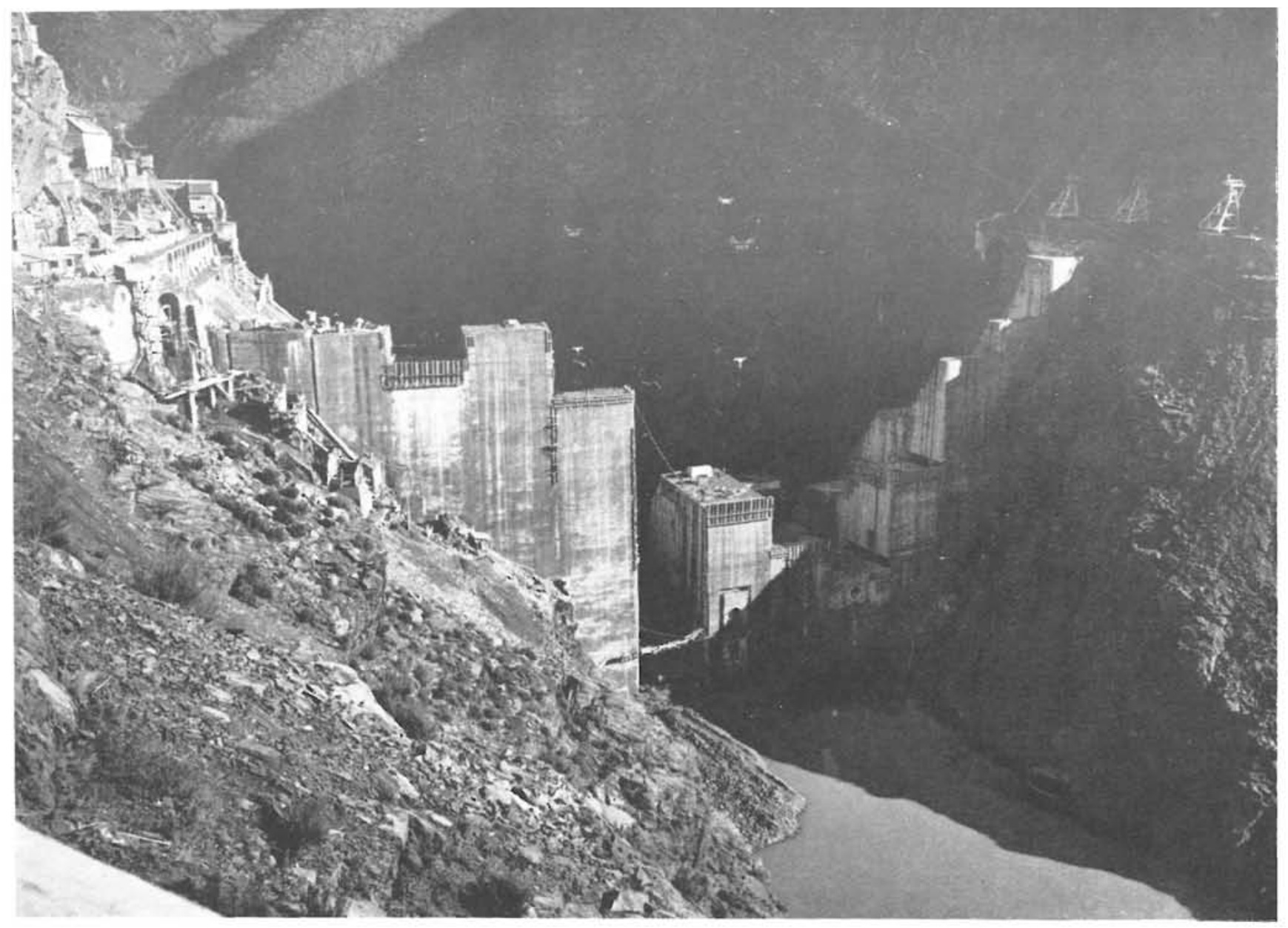

Vista-desde aguas arriba de la presa-, en la que se puede apreciar el portillo que se ha dejado entre bloque para prevenirse contra posibles avenidas. La presa saliendo de eimientos deja emerger
los bloques del hormigonado. En primer plano, los bloques del hormigonado. En primer plano,

\section{Generalidades}

Para el aprovechamiento hidroeléctrico del río Navia. que atraviesa la parte nccidental de la provincia asturiana. se han mancomunado los esfuerzos de la Hidroeléctrica del Cantábrico y la Electra del Viesgo.

En la cabecera del río se ha previsto la construcción de una presa de regulación de caudales. Aguas abajo de esta presa se ha construido el conocido salto de Grandas de Salime; a unos $35 \mathrm{~km}$ más al Norte se halla la presa de Doiras, actualmente en explotación, $y$, finalmente, la presa de Arbón, próxima a la desembocadura del río en el mar, que constituye la primera fase del aprovechamiento del rio Navia.

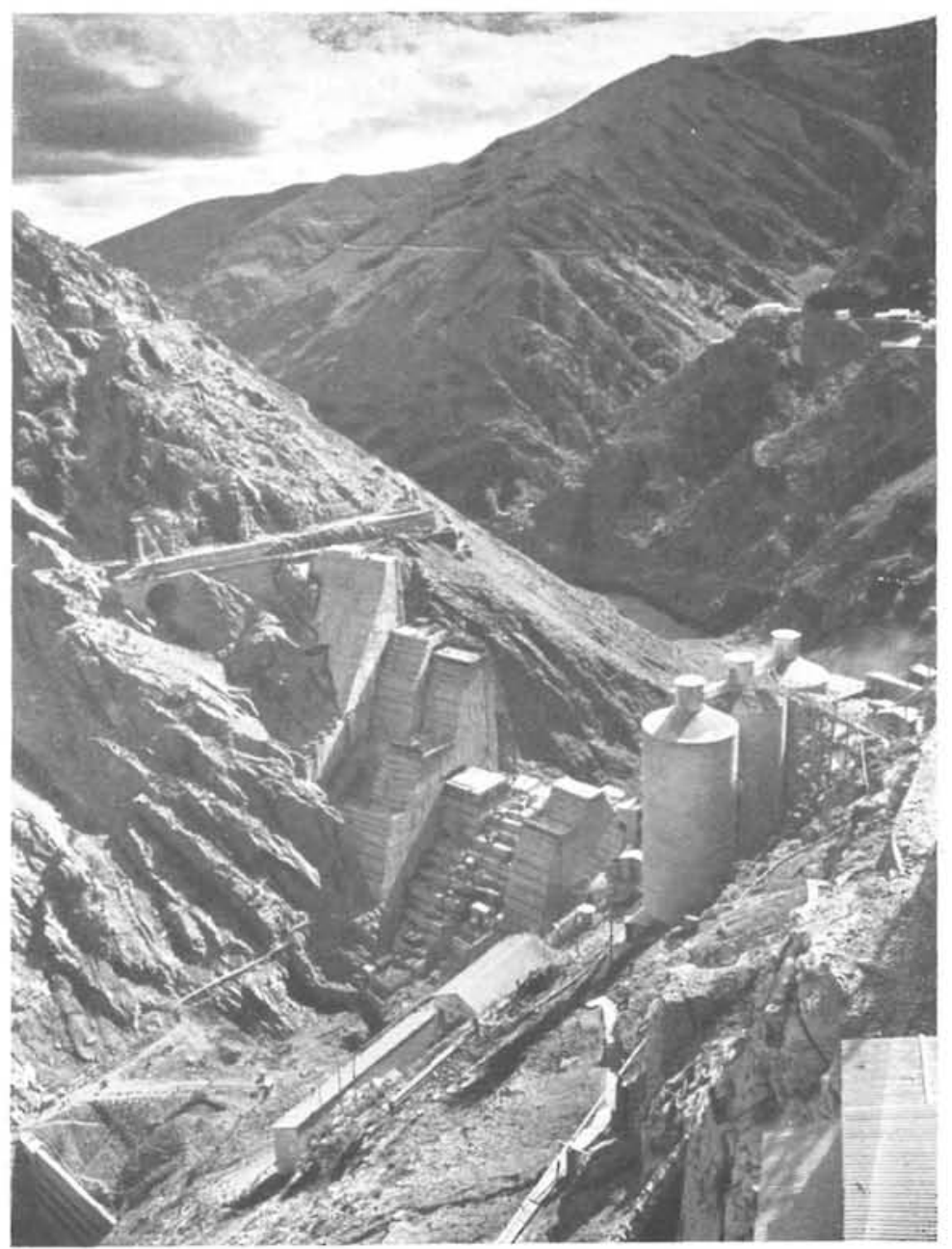




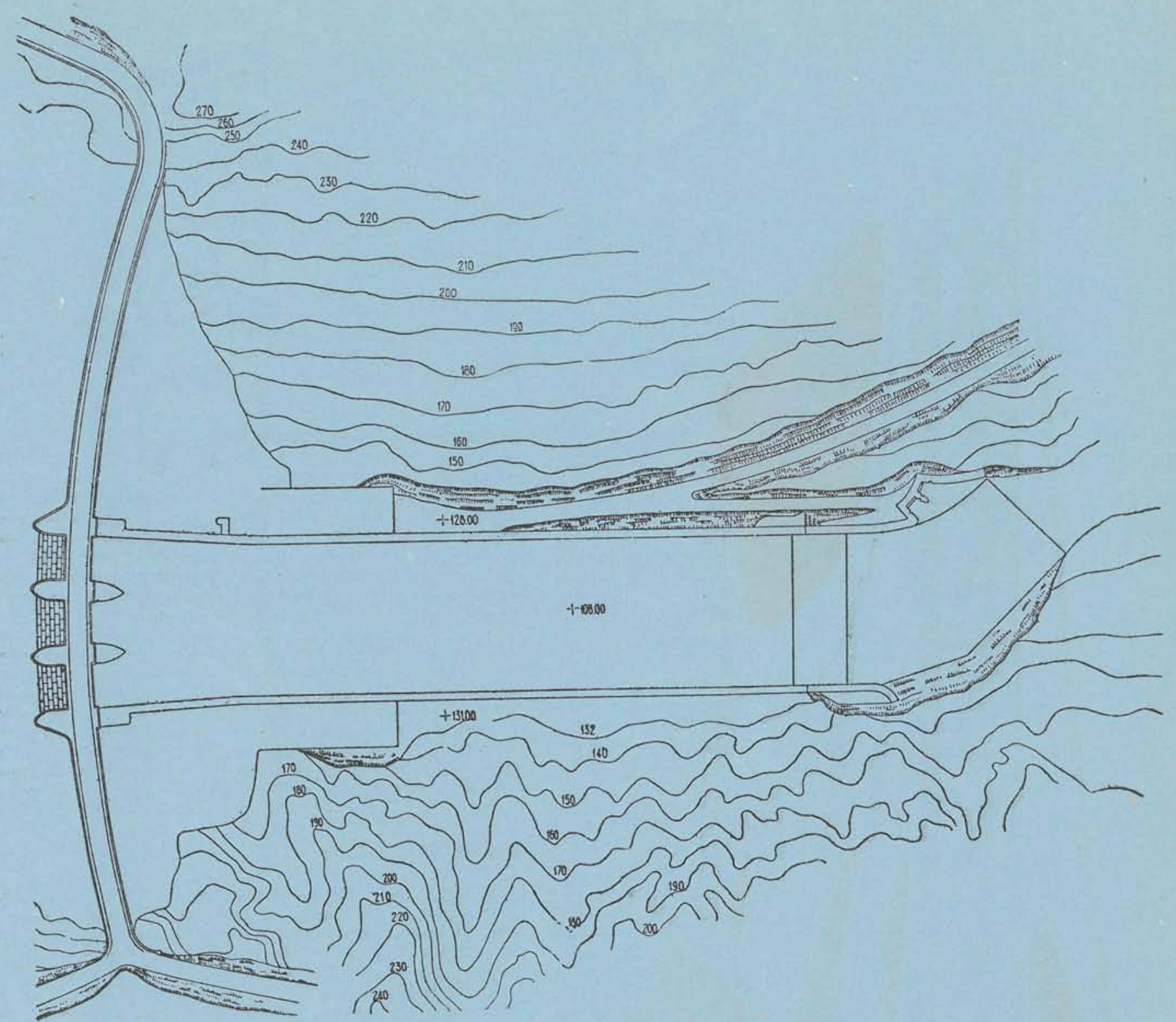

Planta de la presa, cuenco de amortiguación $y$ azud que limita al cuenco.






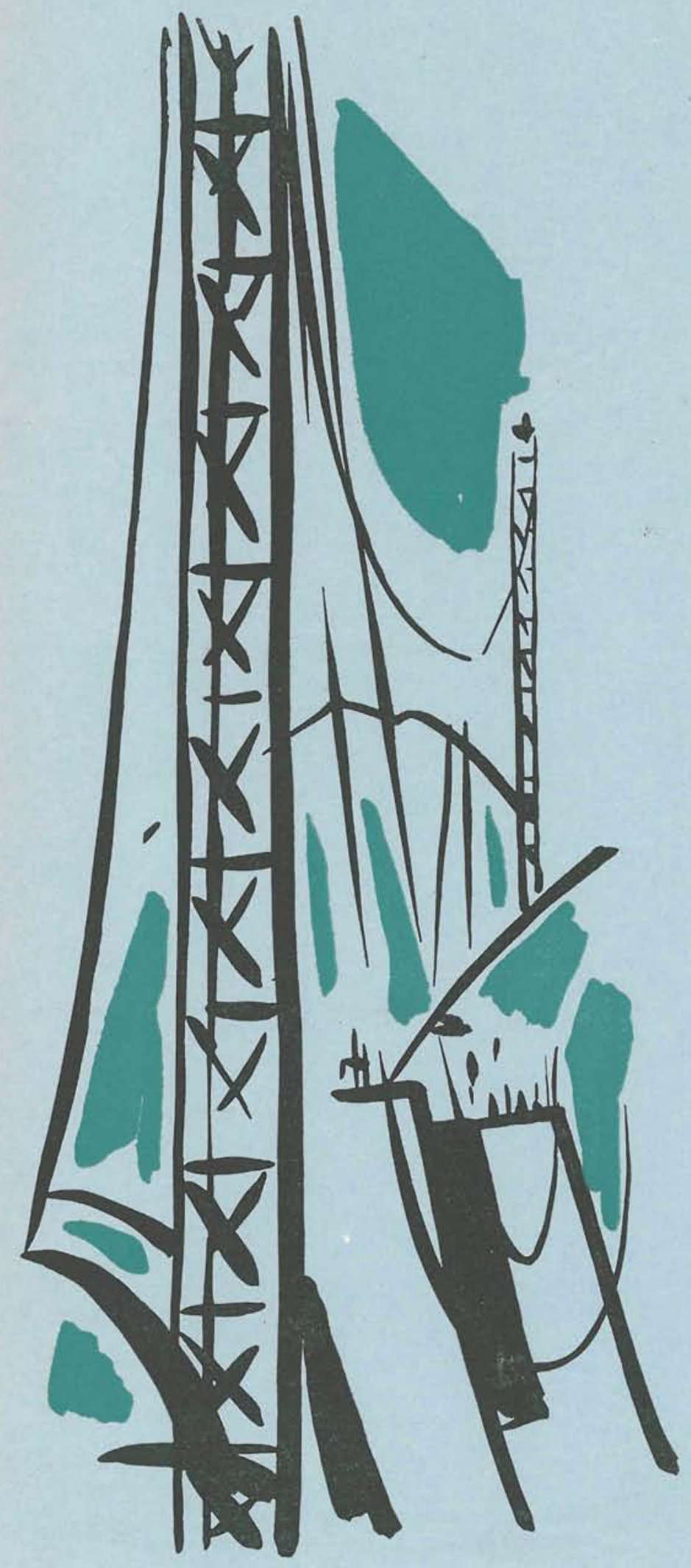

De estos cuatro aprovechamientos parciales del rio destaca, por su volumen, altura y capacidad de produccíon, la presa de Grandas de Salime, a la que en este trabajo se presta y centra el interés dominante.

El salto ha recibido la denominación de Grandas de Salime por hallarse situado en las proximidades del ayuntamiento del mismo nombre. En estos parajes, el cauce del río se encuentra muy encajado, las cerradas se prestan a la construcción de presas de retención, y, aunque el volumen de aguas embalsadas no es de excesiva importancia, se espera obtener un gran rendimiento en la producción de energía eléctrica.

\section{Insfalaciones auxiliares}

Dado el aislamiento del lugar de los centros de habitación y comunicaciones, las instalaciones y obras auxiliares han sido de consideración. Aparte de las instalaciones clásicas ineludibles de este tipo de obras, tales como las correspondientes a los aglomerantes, áridos, campamentos, planos inclinados, transformación, talleres, almacenes, etc., se llevó un gran esfuerzo para poderse comunicar por carretera con Navia, puerto que se pensó utilizar para los grandes sprovisionamientos.

Este último centro se unió a las obras por medio de un cable transportador aéreo, de $40 \mathrm{t}$ de capacidad horaria, que complementaría los transportes por carretera. Como esta linea tiene unos $40 \mathrm{~km}$ de longitud, se subdividió en varios tramos automotores y una estación central de carga y descarga, que se utilizaria para no interrumpir el servicio parcial entre dicha estación y la obra o puerto en caso de avería de una de estas dos partes en que se dividió la línea.

\section{Derivación del río}

Con objeto de poder construir la presa en seco, se procedio - desviar el río, por medio de una pequeña ataguía que derivaba las aguas a una galeria, de $600 \mathrm{~m}$ de longitud y de $200 \mathrm{~m}^{2}$ por segundo de capacidad de desaglle. Aguas arriba del lugar de restitución de aguas de la galería de derivación, se construyó una ataguia para evitar que las aguas llegasen al lugar de la obra. Estas obras auxiliares permitieron dejar en seco al pie de la cerrada elegida para la construcción de la presa.

\section{La presa}

El tipo de presa elegido es de gravedad, formando un arco de $400 \mathrm{~m}$ de radio; tiene $135 \mathrm{~m}$ de altura a partir de cimientos, $92,40 \mathrm{~m}$ de espesor en la base y $8 \mathrm{~m}$ en coronación para formar la plataforma de la carretera que pasa sobre ella; central de pie de presa, y vertedero central. La longitud de la presa es de $280 \mathrm{~m}$ y la toma de agua de la central, en ella situada, se halla de tal forma, que el nivel mínimo aprovechable se encuentra \& $66 \mathrm{~m}$ sobre la base de cimientos; el talud de aguas arriba es de $0,05, y$ el de aguas abajo, de 0,74 . En el interior de la presa se han dejado cuatro galerías de inspección y drenaje, así como una cámara de compuertas para regular el paso de agua.

Esta enorme masa de hormigón cerrará el paso al río para formar un gran embalse, de unos $30 \mathrm{~km}$ de longitud, que se extenderá aguas arriba de la presa y almacenará unos 270 millones de metros cúbicos de agua cuando el nivel de éste, delante de la presa, sea el normal previsto para la explotación. Para dar paso a las aguas sobrantes y grandes avenidas, se ha construído un aliviadero, de $2.000 \mathrm{~m}^{3} / \mathrm{s}$ de capacidad, regulado por medio de compuertas metálicas de sector, de $15 \times 6 \mathrm{~m}$ cada una. 


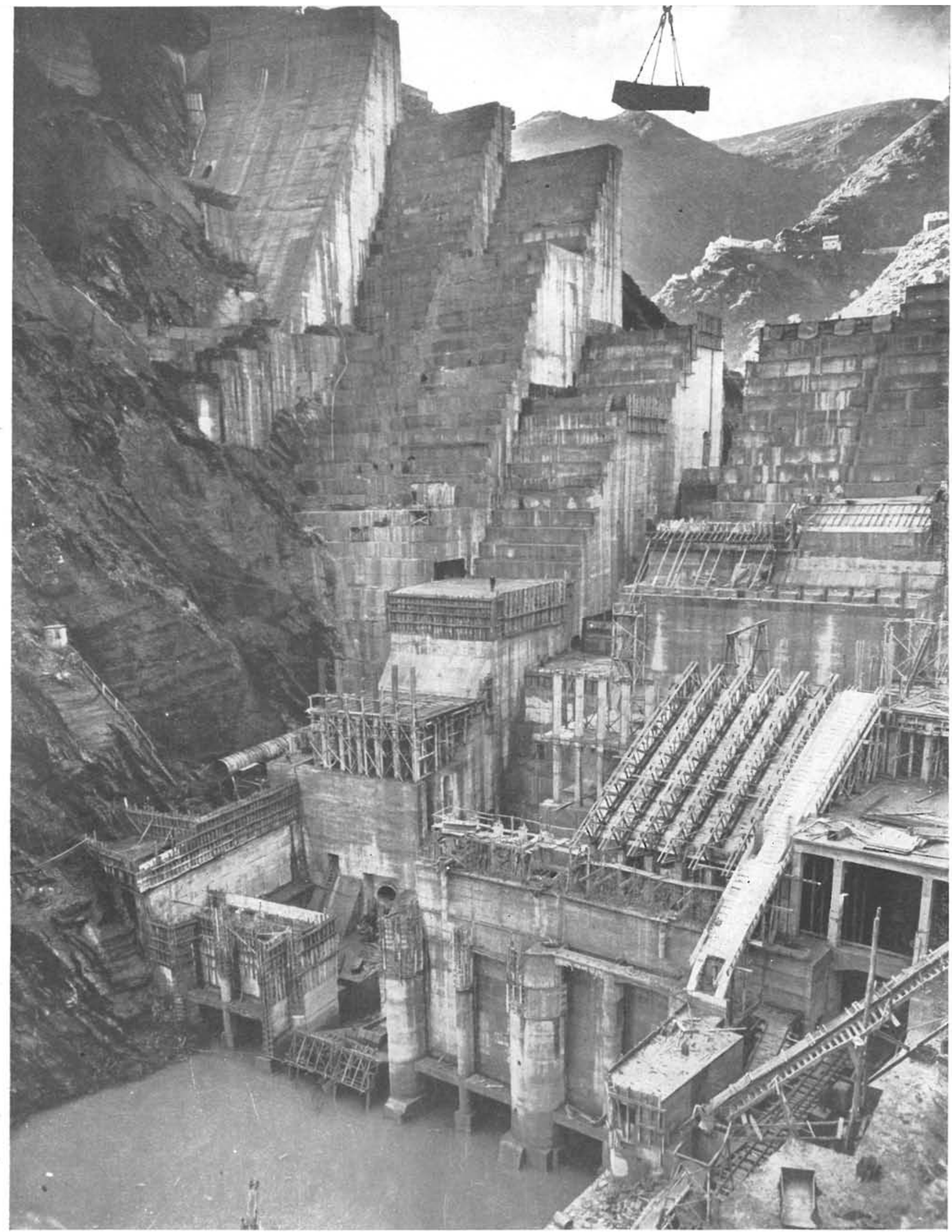

Un momento de la construcción de la presa. 

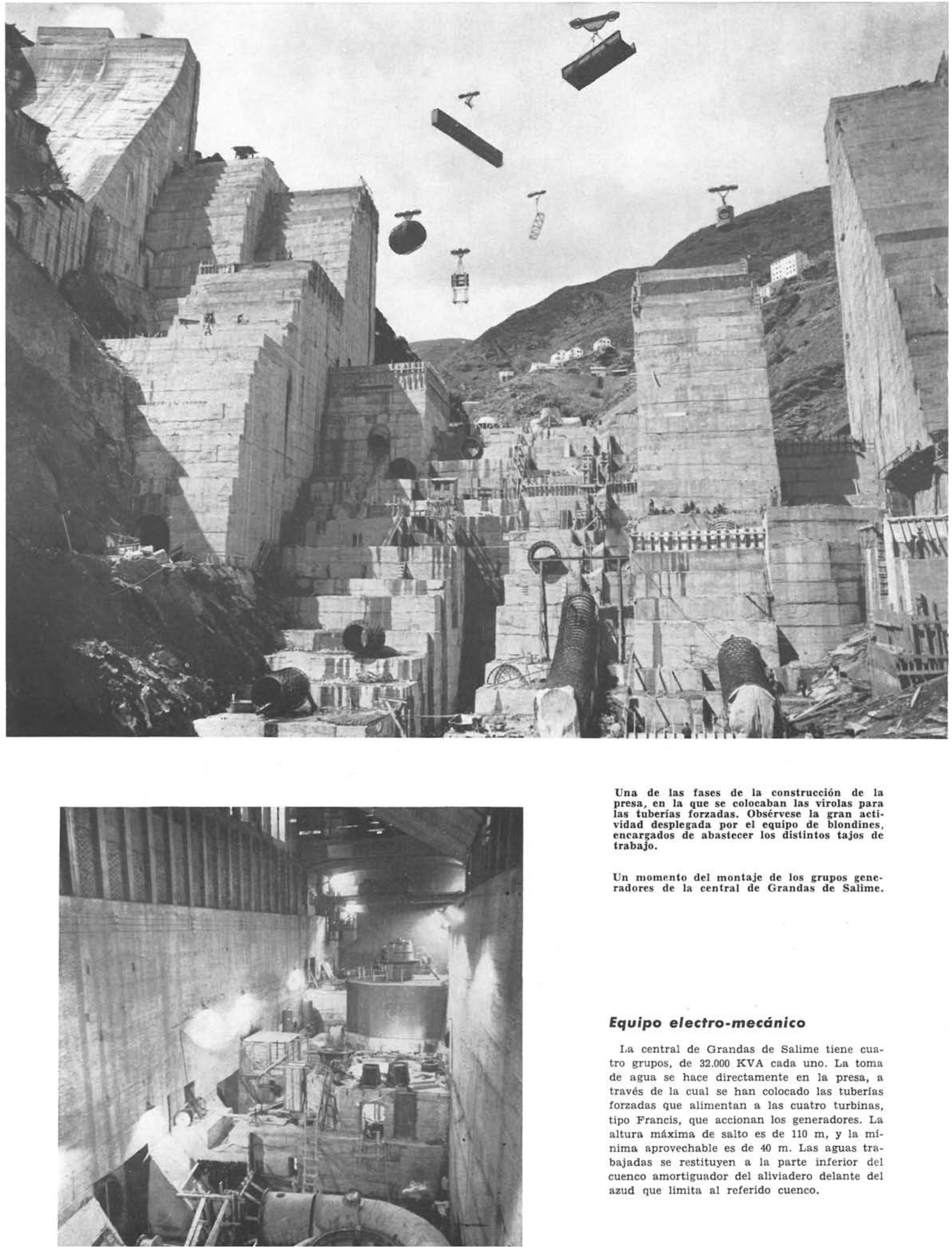

Una de las fases de la construcción de la presa, en la que se colocaban las virolas para las tuberías forzadas. Obsérvese la gran actividad desplegada por el equipo de blondines, encargados de abastecer los distintos tajos de trabajo.

Un momento del montaje de los grupos generadores de la central de Grandas de Salime.

\section{Equipo electro-mecánico}

La central de Grandas de Salime tiene cuatro grupos, de 32.000 KVA cada uno. La toma de agua se hace directamente en la presa, a través de la cual se han colocado las tuberías forzadas que alimentan a las cuatro turbinas, tipo Francis, que accionan los generadores. La altura máxima de salto es de $110 \mathrm{~m}$, y la minima aprovechable es de $40 \mathrm{~m}$. Las aguas trabajadas se restituyen a la parte inferior del cuenco amortiguador del aliviadero delante del azud que limita al referido cuenco. 

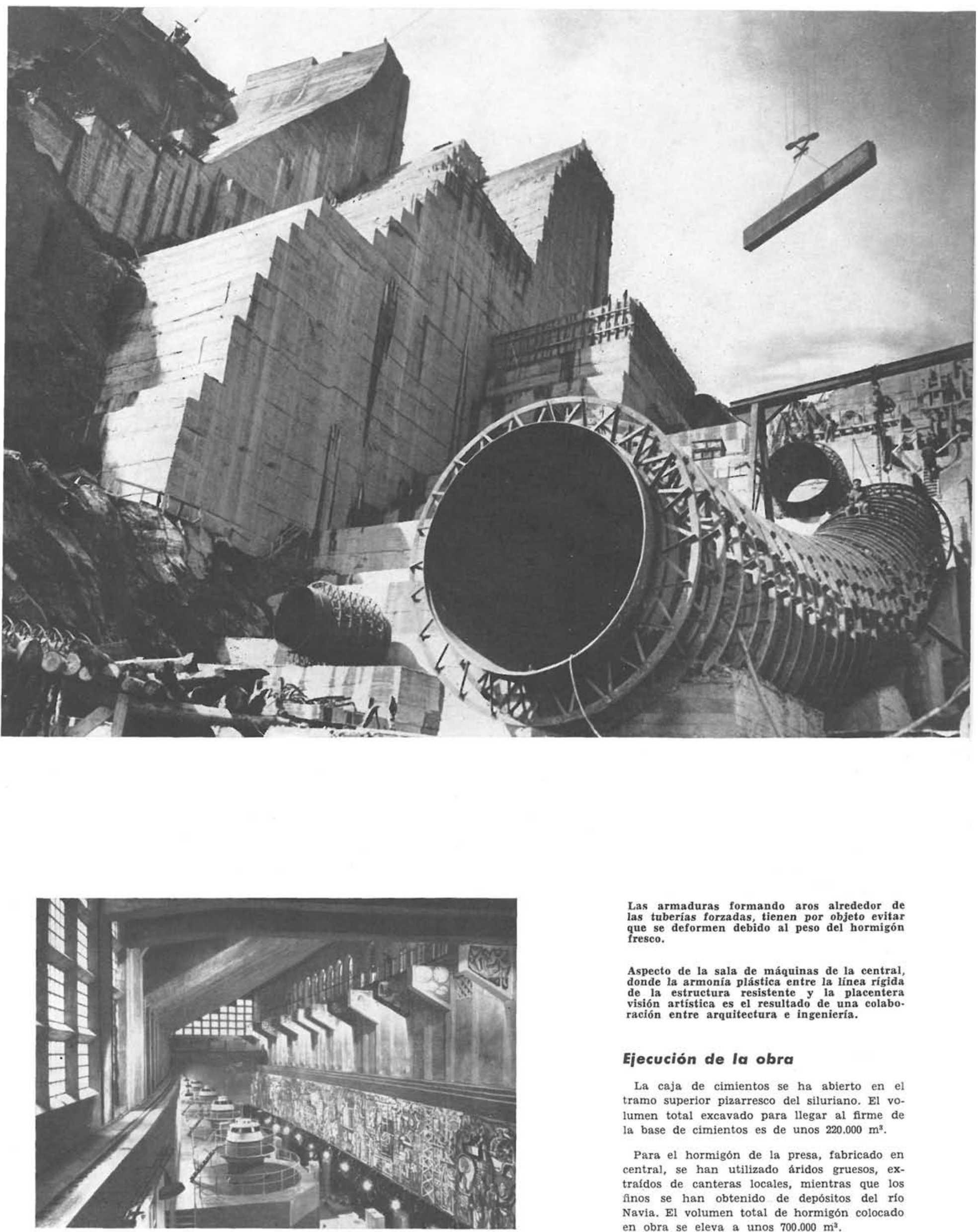

Las armaduras formando aros alrededor de las tuberías forzadas, tienen por objeto evitar que se deformen debido al peso del hormigón

Aspecto de la sala de máquinas de la central, donde la armonía plástica entre la línea rígida de la estructura resistente y la placentera ración entre arquitectura e ingeniería.

\section{Ejecución de la obra}

La caja de cimientos se ha abierto en el tramo superior pizarresco del siluriano. El volumen total excavado para llegar al firme de la base de cimientos es de unos $220.000 \mathrm{~m}^{3}$.

Para el hormigón de la presa, fabricado en central, se han utilizado áridos gruesos, extraídos de canteras locales, mientras que los finos se han obtenido de depósitos del río Navia. El volumen total de hormigón colocado en obra se eleva a unos $700.000 \mathrm{~m}^{3}$. 

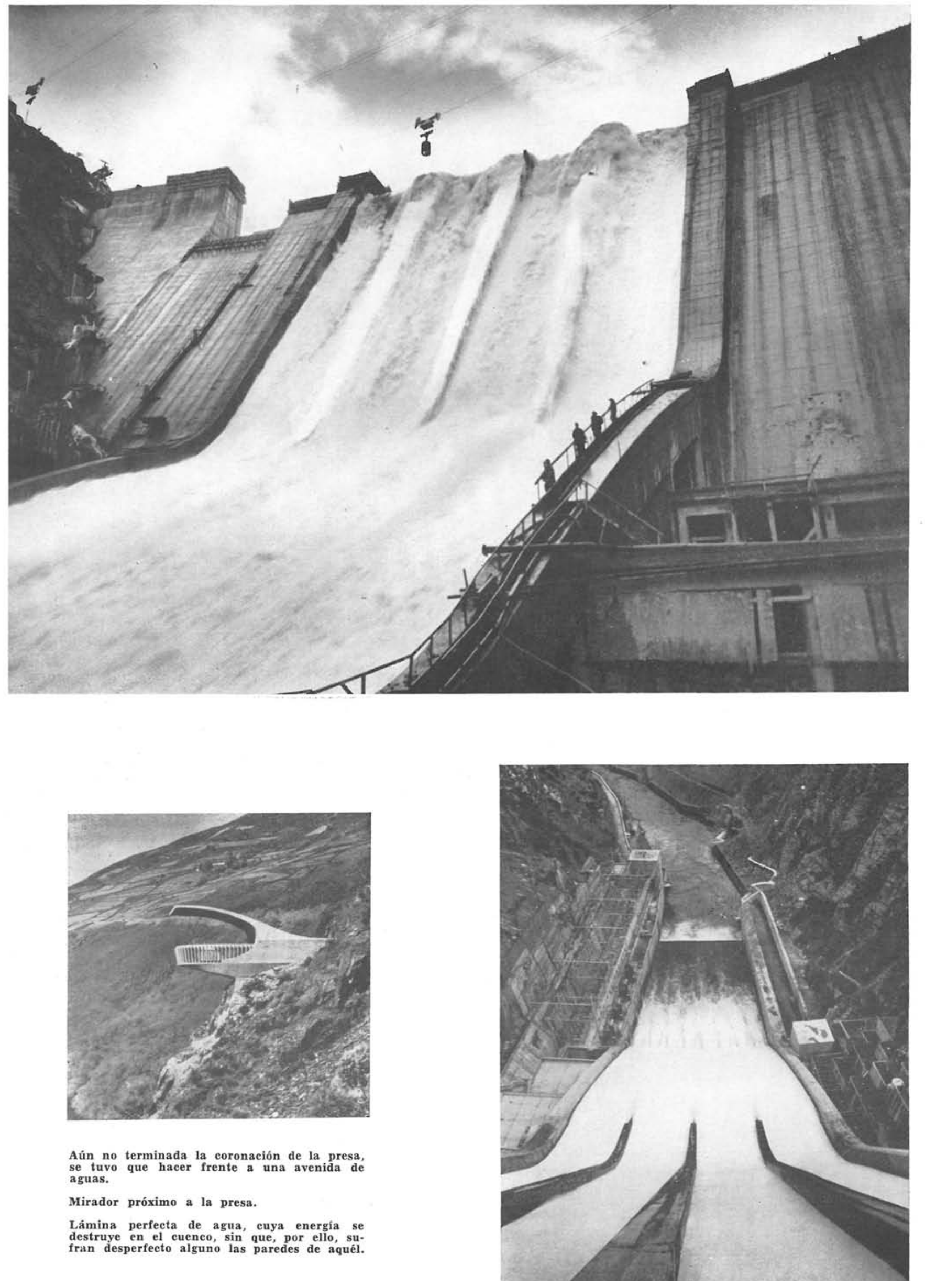

Aún no terminada la coronación de la presa se tuvo que hacer frente a una avenida de aguas.

Mirador próximo a la presa.

Lamina perfecta de agua, cuya energía se fran desperfecto alguno las paredes de aquél. 


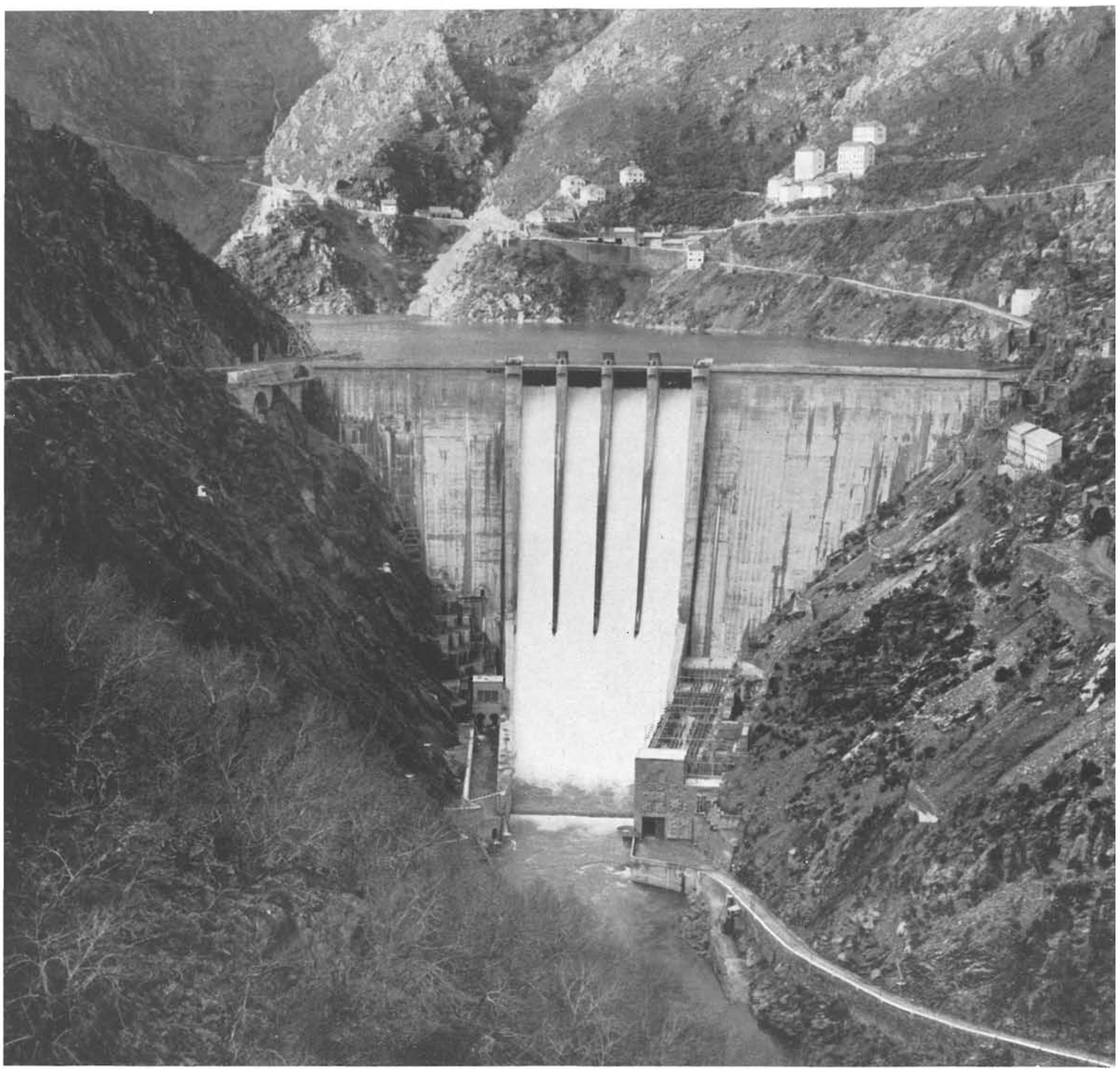

Vista de la presa terminada, desde aguas abajo.
El hormigonado se realizó formando grandes bloques, a los que se les hacía llegar los nateriales por medio de siete blondines convenientemente instalados. Para prevenirse contra los efectos de la retracción se proyectaron juntas de dirección radial; y para la impermeabilización, juntas de chapas de cobre.

La celeridad en el ritmo del hormigonado fué aumentando gradualmente hasta lograr una cadencia del orden de $3.400 \mathrm{~m}^{3}$ por día, volumen que rebasa los rendimientos de otras obras similares europeas, las cuales han empleado una gran mecanización y maquinaria auxiliar. 
La realización de este proyecto, cuyo coste total se ha estimado en unos 500 millones de pesetas, ha constituído un éxito que no se puede regatear, no ya por sus notables características intrínsecas, sino por el gran esfuerzo llevado a cabo por un grupo de personal experto que, desde un principio, tuvo que vencer y ordenar las múltiples soluciones y problemas que toda obra de esta naturaleza y volumen plantea.

J. J. U.

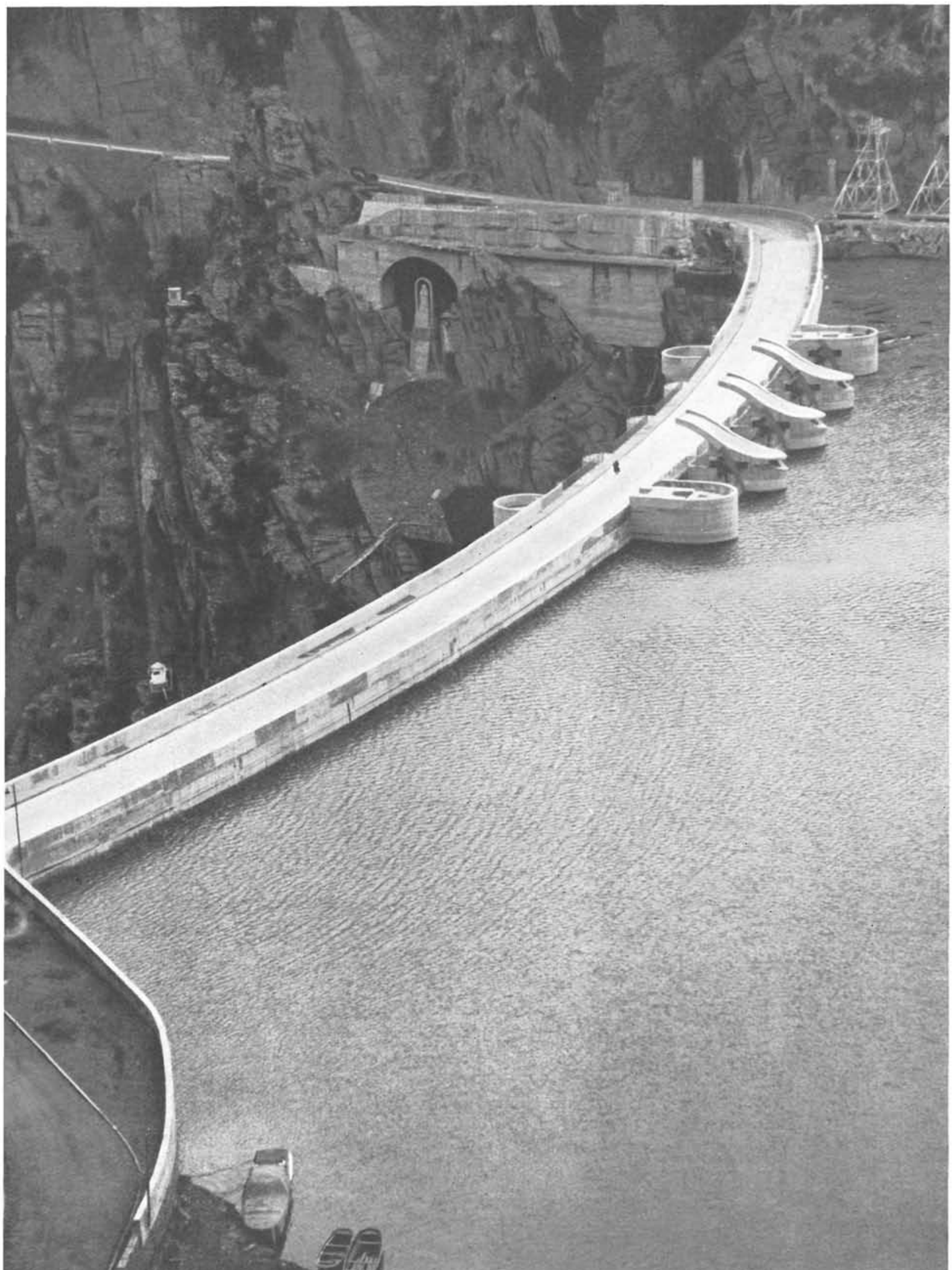

